\title{
Effect of Low-level Laser Therapy on Neurosensory Disorders Recovery following the Bilateral Sagittal Split Ramus Osteotomy
}

\author{
${ }^{1}$ Mohamad T Kiani, ${ }^{2}$ Bahman Derakhshan, ${ }^{3}$ Mina Abasi, ${ }^{4}$ Shahrooz Shafahifard, ${ }^{5}$ Mohammad J Kharazifard
}

\begin{abstract}
Introduction: Bilateral sagittal split osteotomy (BSSO) is a technique commonly used to correct mandibular disproportions, while neurosensory disorders are common following this technique. Low-level laser irradiation has shown promising results to relieve the neurosensory disorders of BSSO technique.
\end{abstract}

Objective: The aim of this study was to compare the effects of low-level laser irradiation on the neurosensory complications related to the BSSO technique.

Materials and methods: In this prospective, double-blinded clinical trial, 13 patients candidate for BSSO surgery were selected and went under complete clinical neurosensory tests (CNTs) including brush stroke discrimination; two-point discrimination; and contact, thermal, and pinprick discrimination, as well as visual analog scale (VAS) assessments. The laser GaAIAs (820-830 $\mu \mathrm{m}$ wavelength) irradiation was done for total six sessions after surgery for the patients at one side accidentally, and the opposite site was a control by irradiation of placebo. The values of CNT assessments between the experimental and control sites were analyzed employing Wilcoxon signed-rank test.

Results: Higher values of VAS and brush stroke discrimination test were observed in the laser-irradiated sites than in the control sites, although without any significant differences $(p>0.05)$. Moreover, significantly higher values of pinprick discrimination test were noted in the experimental sites on days $1,2,3,4,7,14,28$, and $60(p<0.05)$. The thermal test results were higher in the study sides, and the differences of both sides were significant on days $2,3,4,7,14$, and $28(p<0.05)$.

Conclusion: In total, GaAlAs low-level laser irradiation after the BSSO surgery results in both subjective and objective improvements regarding the time and magnitude of return of function; however, in some tests no significant differences were found between laser-irradiated and control areas.

\footnotetext{
1,2,4 Assistant Professor, ${ }^{3}$ Dentist, ${ }^{5}$ Consultant

${ }^{1-3}$ Department of Oral and Maxillofacial Surgery, School of Dentistry, Tehran University of Medical Sciences, Tehran, Islamic Republic of Iran

${ }^{4}$ Department of Oral and Maxillofacial Surgery, School of Dentistry, Islamic Azad University, Tehran, Islamic Republic of Iran

${ }^{5}$ Department of Epidemiology, School of Dentistry, Tehran University of Medical Sciences, Tehran, Islamic Republic of Iran

Corresponding Author: Mohamad T Kiani, Assistant Professor Department of Oral and Maxillofacial Surgery, School of Dentistry Tehran University of Medical Sciences, Tehran, Islamic Republic of Iran, Phone: +00982122024099, e-mail: mokiani@yahoo.com
}

Keywords: Bilateral sagittal split osteotomy, Low-level laser, Neurosensory disorders.

How to cite this article: Kiani MT, Derakhshan B, Abasi M, Shafahifard S, Kharazifard MJ. Effect of Low-level Laser Therapy on Neurosensory Disorders Recovery following the Bilateral Sagittal Split Ramus Osteotomy. Int J Laser Dent 2015;5(2):43-49.

Source of support: Nil

Conflict of interest: None

\section{INTRODUCTION}

Nowadays, bilateral sagittal split ramus osteotomy (BSSRO) has become the most popular technique for correction of mandibular deformities. ${ }^{1}$ Neurosensory deficits of inferior alveolar nerve (IAN) is inevitable complications after this surgery. Incidence of immediate and long-standing neurosensory injuries has been reported dispersal due to the role of the variety of interfering factors, including the variation in surgeons' techniques and expertise, lack of standardization of neurosensory tests, ${ }^{2,3}$ and diversity of different neurosensory test periods. Karas et $\mathrm{al}^{4}$ examined six patients receiving bilateral sagittal split osteotomy (BSSO) surgery and have reported the incidence of the neurosensory disorder among these patients to be $72 \%$ immediately after the surgery, $50 \%$ after 1 month, $25 \%$ after 3 months, and $16 \%$ after 6 months. Some parameters, such as the movement direction of the mandible (retrusion or protrusion), magnitude of mandibular movement, inappropriate osteotomy, IAN manipulation during the surgery, simultaneous 3rd molar tooth surgery and the use of rigid internal fixation instead of intermaxillary fixation, and increase of patients' age are associated with the increase of neurosensory disorders following BSSO surgery. Although some variables related to the incidence of neurosensory disorders can be prevented, many of them cannot be controlled; hence, the injuries to the IAN and the paresthesia associated with it are one of the accepted risks of BSSO surgery. ${ }^{5-7}$

Low-level laser at wavelengths of 820 to $830 \mu \mathrm{m}$ (GaAlAr) has been advocated as one of the peripheral therapeutic options for the treatment of trigeminal nerve paresthesia. ${ }^{8}$ Furthermore, low-level laser is reported to improve the long-standing sensory disorders of IAN. ${ }^{9}$ 
Simple and convenient application and lack of any contraindications, and any possible complications, are only some of the benefits of low-level laser therapy. To penetrate to the intended tissue, the laser energy was scattered several times and distributed to different sites. Absorption of this energy stimulates enzyme activity or prevents it. By this manner, low-level laser passes through the tissue layers and encounter to cell chromophores like mitochondria. Then by activation of enzymes increases the ability of their function for several times. In addition, photochemical reactions lead to the induction of some reactions and physiological processes with some therapeutic effects. In doing so, the laser relieves inflammation and reinforces the immune system through the application of broad therapeutic effects. ${ }^{10}$

Given the significance of this issue, the present study was conducted with the aim of examining the effects of low-level laser therapy on sensory complications resulting from BSSRO surgery.

\section{MATERIALS AND METHODS}

In a prospective double-blinded randomized clinical trial, 13 candidate patients for BSSRO surgery were included in the study for meticulous examination. Inclusion criteria were having mandibular prognathism, not receiving simultaneous genioplasty or other osteotomy treatments, and not suffering from any systemic and neurologic diseases.

Thorough clinical neurosensory tests (CNTs) were conducted for all patients prior to the treatment by a medic who had not played any role in the surgeries. These tests consisted of level A test (brush stroke directional discrimination test and two-point discrimination test), level B test (contact detection), and level C test (pinprick nociception and thermal discrimination). Visual analog scale (VAS) was also employed to determine the subjective neurosensory disorders evaluations.

Clinical neurosensory test was done in an area of $1 \mathrm{~cm}$ in bilateral labiomental site on the basis of Zuniga et $\mathrm{al}^{3}$ protocol. Three different levels of the test were performed on each patient while his eyes were closed. The specific requirements of the neurosensory tests were clarified for the patients; however, to ensure about the patients' thorough understanding of the test, prior to conducting the main IAN test, the test was done on study sites and different other sites, such as hands and arms.

A brush No. 2 was moved within the testing site and in the anterior or posterior directions to carry out level A test with the aim of detecting brush movement directions. To detect the movement direction of the brush, positive responses of 10 tries were recorded. A Boley gauge with blunt tips for evaluating two-point discrimination was applied, and the distances in millimeters were recorded.

Evaluation of contact discrimination test at level B was done by means of Semmes-Weinstein monofilaments (Stoelting Co. Wood Dale, IL, USA). Plastic monofilaments with different sizes were used in this system which were connected to the Lucite rods. Rods and filaments were used with the scales of 3 to 10, which were compatible with the logarithms base 10 in terms of the power (in $\mathrm{mg}$ ) required for folding or bending a single filament of fiber. The minimum bending force for rods and filaments - terms of grams, at the level that patient was continuously able to detect - were recorded.

Level $C$ test included thermal detection and was done using sprayed ethyl chloride on an applicator with cotton tip compared with the other applicator with dried cotton tip. The patients were asked to provide their responses to the question whether they feel the cold and moisture while using ethyl chloride. If correct responses were provided in at least 7 out of 10 times of test replication, test value was considered to be positive.

Subjective assessments of neurosensory disorders were made using $10-\mathrm{cm}$ VAS by 5 scales and $2.5-\mathrm{cm}$ intervals. Visual analog scale division contains 1 (complete absence of sense), 2 (approximate absence of sense), 3 (reduced sense), 4 (approximate normal sense), and 5 (full normal sense). The patients were requested to mark on the line on the basis of their perception at various testing sessions. The distance was measured and recorded along the line. The mean value of CNT and VAS test results were specified for each course of time. Furthermore, a linear model was used for the overall evaluation of tests.

In level $C$ test, the pin pain tester was done employing the pin, and the results were compared using the applicator with cotton tip. The patients were inquired to inform any sense of sharpness or heaviness during the test. In the case of providing correct responses in 7 out of 10 times of conducting the test, test value was considered to be positive.

All patients were treated by one surgeon in order to set back the mandible with using the BSSRO technique while the mandible was fixed in each side using two titanium screws.

Laser irradiation protocol was also performed on the basis of Khullar et $\mathrm{al}^{9}$ study.

To do so, GaAlAr diode low-level laser (photon plus gallium-aluminum arsenide diode), along with a control console and handheld probe connected to a cable, was used in this study (Ronvig A/S, Vejle, Denmark). Probe diameter at the laser irradiation point was $18 \mathrm{~mm}$, laser irradiation was $70 \mathrm{~mW}$, and the wavelength was within the range of 830 to $820 \mu \mathrm{m}$ (close to the infrared 
spectrum). Laser spot size was $1.3 \mathrm{~cm}^{2}$, its power density was $550 \mathrm{~mW} / \mathrm{cm}^{2}$, and the medical device applied 6/0 J energy in any treatment site. Laser probe was directly used in the treatment sites and a gel was used between the probe and the skin surface for the outer oral sites. In the inner oral cavity sites, salivary played the role of conductive environment; hence, the majority of patients had no sense during the laser irradiation.

A beep-like sound was heard at the beginning and end of the treatment which lasted approximately 90 seconds in each site. Hence, one treatment session for the four sites conducted bilaterally lasted about 12 minutes. Actual low-level laser therapy was conducted (laser devices was prepared for irradiation in the control sites; however, laser irradiation was not activated) with $0 / 6 \mathrm{~J}$ energy in each session and along the IAN in four sites covering the left and right sides of the mandible. Outer oral cavity treatment sites included lower lip and the chin (cutaneous distribution of mental nerve), and the inner oral cavity sites included the mental foramen on the buccal vestibule of the mandible between the apex of the 1st and 2nd premolars teeth, osteotomy site in the buccal vestibule of mandible at the apex of the mandible's 2nd molar tooth, and mental foramen on the lateral raphe of pterygomandibular. In total and after the surgery, six sessions on days 1, 2, 3, 4, 7, and 14 were hold; no irradiation has been done on days 14, 28, and 60; and only CNT and VAS parameters were evaluated on those days.

Mean, standard deviation, minimum and maximum values of the results, as well as the values of different CNTs on different days and for different sites under the lowlevel laser irradiation and those under no low-level laser irradiation were determined and reported. The obtained results of various neurosensory tests on different days

Table 1: Central dispersion indicators of two-point discrimination test values for the laser-irradiated and control sides on different days

\begin{tabular}{llllll}
\hline Direction and day & Number & Mean & SD & Minimum & Maximum \\
\hline Irradiation: Day 1 & 13 & $13 / 54$ & $1 / 39$ & $11 / 0$ & $16 / 0$ \\
Control: Day 1 & 13 & $14 / 0$ & $1 / 41$ & $12 / 0$ & $16 / 0$ \\
Irradiation: Day 2 & 13 & $13 / 46$ & $1 / 33$ & $11 / 0$ & $16 / 0$ \\
Control: Day 2 & 13 & $13 / 92$ & $1 / 61$ & $12 / 0$ & $16 / 0$ \\
Irradiation: Day 3 & 13 & $12 / 78$ & $1 / 29$ & $11 / 0$ & $15 / 0$ \\
Control: Day 3 & 13 & $13 / 31$ & $1 / 6$ & $11 / 0$ & $17 / 0$ \\
Irradiation: Day 4 & 13 & $12 / 35$ & $1 / 07$ & $11 / 0$ & $14 / 0$ \\
Control: Day 4 & 13 & $12 / 58$ & $1 / 71$ & $10 / 0$ & $16 / 0$ \\
Irradiation: Day 7 & 13 & $10 / 08$ & $1 / 04$ & $8 / 0$ & $12 / 0$ \\
Control: Day 7 & 13 & $10 / 96$ & $1 / 92$ & $8 / 0$ & $14 / 0$ \\
Irradiation: Day 14 & 13 & $9 / 62$ & $0 / 96$ & $8 / 0$ & $11 / 0$ \\
Control: Day 14 & 13 & $10 / 62$ & $1 / 98$ & $8 / 0$ & $15 / 0$ \\
Irradiation: Day 28 & 13 & $9 / 23$ & $0 / 93$ & $8 / 0$ & $11 / 0$ \\
Control: Day 28 & 13 & $10 / 04$ & $1 / 59$ & $8 / 0$ & $13 / 0$ \\
Irradiation: Day 60 & 13 & $8 / 85$ & $0 / 89$ & $8 / 0$ & $10 / 0$ \\
Control: Day 60 & 13 & $8 / 92$ & $0 / 86$ & $8 / 0$ & $11 / 0$ \\
\hline
\end{tabular}

were compared between the experimental and control groups using the Wilcoxon signed-ranks test.

\section{RESULTS}

Overall, the results of two-point discrimination test were slightly higher in the control side throughout all days in comparison to the results obtained from the laserirradiated side, although the differences between two sides were not significant in any of the days [on days 1 , 2, 3, 4, 7, 14, 28, 60 ( $\mathrm{p}>0.05$ ) Table 1].

The obtained results of pinprick test was significantly higher for the low-level laser-irradiated side on days 1, 2, $3,4,7,14,28$ and 60 ( $\mathrm{p}<0.05)$ in comparison to those of the control side (Table 2).

Throughout all days of irradiation, the results of brush stroke discrimination test was slightly higher for the experimental group compared with the control one, although no significant difference was observed between two sides on days $1,2,3,4,7,14,28$, and 60 (p > 0/05) Table 3).

Throughout all days, thermal test values of laserirradiated sites were higher than those of the sites without irradiation. Although the differences were not significant on days $1(p=0 / 1)$ and $60(p=0 / 1)$, significant differences were observed in two groups' values of thermal test on days $2,3,4,7,14$, and 28 ( $\mathrm{p}<0.05)$. For more details, refer to Table 4.

Although the mean values of VAS in the experimental group's sites were slightly higher than those of the control group throughout all days, these differences were not significant in any of the days on the basis of Wilcoxon signed-rank test [days 1, 2, 3, 4, 7, 14, 28, and 60 ( $p>0.05)$ ]. Table 5 presents more details.

Table 2: Central dispersion indicators of pinprick test results for laser-irradiated and control sides on different days

\begin{tabular}{llllll}
\hline Direction and day & Number & Mean & SD & Minimum & Maximum \\
\hline Irradiation: Day 1 & 13 & $6 / 23$ & $1 / 48$ & $4 / 0$ & $8 / 0$ \\
Control: Day 1 & 13 & $5 / 38$ & $1 / 33$ & $3 / 0$ & $7 / 0$ \\
Irradiation: Day 2 & 13 & $6 / 69$ & $1 / 32$ & $5 / 0$ & $9 / 0$ \\
Control: Day 2 & 13 & $6 / 08$ & $1 / 26$ & $4 / 0$ & $8 / 0$ \\
Irradiation: Day 3 & 13 & $7 / 31$ & $1 / 25$ & $5 / 0$ & $9 / 0$ \\
Control: Day 3 & 13 & $6 / 62$ & $1 / 12$ & $5 / 0$ & $8 / 0$ \\
Irradiation: Day 4 & 13 & $7 / 69$ & $0 / 95$ & $6 / 0$ & $9 / 0$ \\
Control: Day 4 & 13 & $7 / 0$ & $1 / 08$ & $5 / 0$ & $8 / 0$ \\
Irradiation: Day 7 & 13 & $9 / 08$ & $0 / 49$ & $8 / 0$ & $10 / 0$ \\
Control: Day 7 & 13 & $8 / 08$ & $0 / 64$ & $7 / 0$ & $9 / 0$ \\
Irradiation: Day 14 & 13 & $9 / 46$ & $0 / 52$ & $9 / 0$ & $10 / 0$ \\
Control: Day 14 & 13 & $8 / 38$ & $0 / 51$ & $8 / 0$ & $9 / 0$ \\
Irradiation: Day 28 & 13 & $9 / 77$ & $0 / 44$ & $9 / 0$ & $10 / 0$ \\
Control: Day 28 & 13 & $9 / 0$ & $0 / 58$ & $8 / 0$ & $10 / 0$ \\
Irradiation: Day 60 & 13 & $9 / 92$ & $0 / 28$ & $9 / 0$ & $10 / 0$ \\
Control: Day 60 & 13 & $9 / 54$ & $0 / 52$ & $9 / 0$ & $10 / 0$ \\
\hline
\end{tabular}


Table 3: Central dispersion indicators of brush strokes test results for laser-irradiated and control sides on different days

\begin{tabular}{llllll}
\hline Direction and day & Number & Mean & SD & \multicolumn{2}{c}{ Minimum Maximum } \\
\hline Irradiation: Day 1 & 13 & $4 / 15$ & $1 / 14$ & $2 / 0$ & $6 / 0$ \\
Control: Day 1 & 13 & $3 / 92$ & $1 / 26$ & $1 / 0$ & $6 / 0$ \\
Irradiation: Day 2 & 13 & $4 / 69$ & $1 / 11$ & $3 / 0$ & $7 / 0$ \\
Control: Day 2 & 13 & $4 / 46$ & $1 / 27$ & $3 / 0$ & $7 / 0$ \\
Irradiation: Day 3 & 13 & $5 / 38$ & $0 / 96$ & $4 / 0$ & $7 / 0$ \\
Control: Day 3 & 13 & $5 / 31$ & $1 / 03$ & $4 / 0$ & $7 / 0$ \\
Irradiation: Day 4 & 13 & $6 / 08$ & $0 / 95$ & $4 / 0$ & $8 / 0$ \\
Control: Day 4 & 13 & $5 / 77$ & $0 / 93$ & $4 / 0$ & $7 / 0$ \\
Irradiation: Day 7 & 13 & $7 / 46$ & $1 / 05$ & $6 / 0$ & $9 / 0$ \\
Control: Day 7 & 13 & $7 / 08$ & $1 / 32$ & $5 / 0$ & $9 / 0$ \\
Irradiation: Day 14 & 13 & $8 / 31$ & $1 / 11$ & $6 / 0$ & $10 / 0$ \\
Control: Day 14 & 13 & $8 / 23$ & $1 / 17$ & $6 / 0$ & $10 / 0$ \\
Irradiation: Day 28 & 13 & $9 / 15$ & $0 / 8$ & $8 / 0$ & $10 / 0$ \\
Control: Day 28 & 13 & $8 / 69$ & $1 / 25$ & $6 / 0$ & $10 / 0$ \\
Irradiation: Day 60 & 13 & $10 / 0$ & 0 & $10 / 0$ & $10 / 0$ \\
Control: Day 60 & 13 & $9 / 46$ & $0 / 88$ & $8 / 0$ & $10 / 0$ \\
\hline
\end{tabular}

Table 5: Central dispersion indicators of VAS test results for laser-irradiated and control sides on different days

\begin{tabular}{llllll}
\hline Direction and day & Number Mean & SD & Minimum & Maximum \\
\hline Irradiation: Day 1 & 13 & $0 / 73$ & $0 / 78$ & 0 & $2 / 0$ \\
Control: Day 1 & 13 & $0 / 69$ & $0 / 72$ & 0 & $2 / 0$ \\
Irradiation: Day 2 & 13 & $2 / 08$ & $0 / 67$ & $1 / 0$ & $3 / 0$ \\
Control: Day 2 & 13 & $2 / 0$ & $0 / 65$ & $1 / 0$ & $3 / 0$ \\
Irradiation: Day 3 & 13 & $3 / 38$ & $0 / 71$ & $2 / 0$ & $4 / 5$ \\
Control: Day 3 & 13 & $3 / 15$ & $0 / 85$ & $1 / 0$ & $4 / 5$ \\
Irradiation: Day 4 & 13 & $4 / 73$ & $0 / 78$ & $3 / 5$ & $6 / 0$ \\
Control: Day 4 & 13 & $4 / 5$ & $0 / 98$ & $3 / 0$ & $6 / 0$ \\
Irradiation: Day 7 & 13 & $6 / 58$ & $1 / 27$ & $5 / 0$ & $8 / 0$ \\
Control: Day 7 & 13 & $6 / 19$ & $1 / 58$ & $3 / 5$ & $9 / 5$ \\
Irradiation: Day 14 & 13 & $7 / 65$ & $1 / 01$ & $6 / 0$ & $0 / 9$ \\
Control: Day 14 & 13 & $7 / 35$ & $1 / 14$ & $5 / 5$ & $9 / 5$ \\
Irradiation: Day 28 & 13 & $8 / 27$ & $0 / 99$ & $6 / 0$ & $10 / 0$ \\
Control: Day 28 & 13 & $7 / 15$ & $2 / 08$ & $1 / 0$ & $9 / 0$ \\
Irradiation: Day 60 & 13 & $8 / 87$ & $0 / 66$ & $8 / 0$ & $10 / 0$ \\
Control: Day 60 & 13 & $7 / 85$ & $2 / 16$ & $1 / 0$ & $9 / 5$ \\
\hline
\end{tabular}

Taking into account the closeness of the mean values of the contact discrimination test and lack of any noticeable differences among them, no significant differences between two groups were observed with respect to the values of contact discrimination test on days 1, 2, 3, 4, 7, 14,28 , and 60 ( $p>0.05$ ). Detailed information is provided in Table 6.

\section{DISCUSSION}

According to the obtained results of the present study, although the mean values of VAS were observed to be slightly higher in laser-irradiated experimental sites throughout all days in comparison to no laser irradiation control sites, the observed differences were not significant in any of the days. Visual analog scale values increased
Table 4: Central dispersion indicators of thermal test results for laser-irradiated and control sides on different days

\begin{tabular}{|c|c|c|c|c|c|}
\hline Direction and day & Number & Mean & $S D$ & Minimum & Maximum \\
\hline Irradiation: Day 1 & 13 & $4 / 92$ & $1 / 04$ & $3 / 0$ & $7 / 0$ \\
\hline Control: Day 1 & 13 & $4 / 62$ & $0 / 96$ & $3 / 0$ & $6 / 0$ \\
\hline Irradiation: Day 2 & 13 & $5 / 92$ & $0 / 95$ & $5 / 0$ & $8 / 0$ \\
\hline Control: Day 2 & 13 & $5 / 23$ & $1 / 01$ & $4 / 0$ & $7 / 0$ \\
\hline Irradiation: Day 3 & 13 & $6 / 38$ & $0 / 87$ & $5 / 0$ & $8 / 0$ \\
\hline Control: Day 3 & 13 & $5 / 92$ & $0 / 95$ & $5 / 0$ & $8 / 0$ \\
\hline Irradiation: Day 4 & 13 & $7 / 15$ & $1 / 07$ & $5 / 0$ & $9 / 0$ \\
\hline Control: Day 4 & 13 & $6 / 23$ & $0 / 93$ & $5 / 0$ & $8 / 0$ \\
\hline Irradiation: Day 7 & 13 & $8 / 69$ & $0 / 63$ & $7 / 0$ & $9 / 0$ \\
\hline Control: Day 7 & 13 & $7 / 92$ & $0 / 86$ & $6 / 0$ & $9 / 0$ \\
\hline Irradiation: Day 14 & 13 & $9 / 31$ & $0 / 63$ & $8 / 0$ & $10 / 0$ \\
\hline Control: Day 14 & 13 & $8 / 31$ & $0 / 63$ & $7 / 0$ & $9 / 0$ \\
\hline Irradiation: Day 28 & 13 & $9 / 54$ & $0 / 66$ & $8 / 0$ & $10 / 0$ \\
\hline Control: Day 28 & 13 & $8 / 77$ & $0 / 73$ & $7 / 0$ & $10 / 0$ \\
\hline Irradiation: Day 60 & 13 & 9/77 & $0 / 44$ & $9 / 0$ & $10 / 0$ \\
\hline Control: Day 60 & 13 & $9 / 46$ & $0 / 66$ & $8 / 0$ & $10 / 0$ \\
\hline
\end{tabular}

Table 6: Central dispersion indicators of contact discrimination test results for laser-irradiated and control sides on different days

\begin{tabular}{llllll}
\hline Direction and day & Number Mean & SD & Minimum & Maximum \\
\hline Irradiation: Day 1 & 13 & $7 / 42$ & $0 / 67$ & $6 / 0$ & $8 / 0$ \\
Control: Day 1 & 13 & $7 / 5$ & $0 / 67$ & $6 / 0$ & $8 / 0$ \\
Irradiation: Day 2 & 13 & $7 / 38$ & $0 / 51$ & $7 / 0$ & $8 / 0$ \\
Control: Day 2 & 13 & $7 / 15$ & $0 / 69$ & $6 / 0$ & $8 / 0$ \\
Irradiation: Day 3 & 13 & $6 / 54$ & $0 / 66$ & $6 / 0$ & $8 / 0$ \\
Control: Day 3 & 13 & $6 / 69$ & $0 / 63$ & $5 / 0$ & $7 / 0$ \\
Irradiation: Day 4 & 13 & $5 / 85$ & $0 / 8$ & $5 / 0$ & $7 / 0$ \\
Control: Day 4 & 13 & $6 / 23$ & $0 / 83$ & $5 / 0$ & $8 / 0$ \\
Irradiation: Day 7 & 13 & $5 / 31$ & $1 / 11$ & $4 / 0$ & $7 / 0$ \\
Control: Day 7 & 13 & $5 / 54$ & $0 / 88$ & $4 / 0$ & $7 / 0$ \\
Irradiation: Day 14 & 13 & $5 / 08$ & $0 / 86$ & $4 / 0$ & $6 / 0$ \\
Control: Day 14 & 13 & $4 / 92$ & $0 / 86$ & $4 / 0$ & $7 / 0$ \\
Irradiation: Day 28 & 13 & $4 / 54$ & $1 / 05$ & $3 / 0$ & $7 / 0$ \\
Control: Day 28 & 13 & $4 / 31$ & $1 / 03$ & $3 / 0$ & $6 / 0$ \\
Irradiation: Day 60 & 13 & $4 / 15$ & $0 / 99$ & $3 / 0$ & $6 / 0$ \\
Control: Day 60 & 13 & $4 / 08$ & $1 / 19$ & $3 / 0$ & $7 / 0$ \\
\hline
\end{tabular}

consistently over time for all the patients in both the control and experimental sides; however, considering the 2-month length of the investigation, no information is available following these 2 months, most likely their neurosensory function has been improved over time. In general, the performance of the laser irradiation, considering the VAS criterion, was evaluated to lead to better results compared with the control group in spite of the observed insignificant differences.

Moreover, values of pinprick test at the laser-irradiated side on all days were found to be significantly higher than those of the control side. With respect to the results of the brush strokes test, slightly higher values were reported for the sites with low-level laser irradiation compared with those of the control sites on all days of irradiation; 
however, it must be mentioned that the difference was not significant on different days.

Furthermore, the results of thermal test in both laserirradiated and control sides revealed that test values of laser-irradiated sites were higher than the values of control sites and the difference was observed to be significant for all days except for days 1 and 60. However, no significant difference was found between the laserirradiated and nonirradiated laser sites with regard to the contact test values. Additionally, the results of two-point discrimination test in both laser-irradiated (experimental) and nonirradiated laser (control) sides showed that the test values in the control side were slightly higher than the laser-irradiated side on all days, though with no significant differences.

All in all, neurosensory disorders of the patients were improved over time in the control and experimental sites; however, recovery was faster in the laser-irradiated side, and the observed difference was significant with respect to a number of parameters (contact and pinprick tests). The obtained results have been highlighted in some of the previously conducted studies.

Khullar et $\mathrm{al}^{9}$ examined the effect of treatment with GaAlAs low-level laser at a wavelength of $820 \mu \mathrm{m}$ during the contact and temperature sensory perception test subsequent to the IAN injuries resulting from the surgery. To address the objectives of the study, 13 patients were selected and assigned to two groups; one of the groups was treated using the actual laser $(4 \times 6 \mathrm{~J})$ in each treatment with IAN distribution during all 20 sessions, while the other group received placebo low-level laser treatment. The results revealed that the actual low-level laser group had significant improvements in their sensory function; however, significant improvements were not noticed for the temperature sensation for any of the groups after the treatment. ${ }^{11}$

Similarly, Khullar et $\mathrm{al}^{9}$ assessed the effect of treatment with GaAlAs low-level laser at a wavelength of $820 \mu \mathrm{m}$ on improving the sensory function of the patients. The obtained results indicated that in both groups, patients had shown relatively the same amounts of VAS values before the treatment for both lips and the chin. However, after completion of the therapy, laser group experienced significant improvements in terms of subjective assessments of Neurosensory Disorders (NSD) scales for both of the lips and the chin on the basis of VAS criterion. The degree of sensory decline of temperature was approximately the same for both groups prior to the treatment, and significant improvement was not observed with respect to the sensory perception between the experimental and control groups. In general, GaAlAs low-level laser irradiation resulted in significant subjec- tive and objective improvements of mechanical sensory perception in the treatment of long-standing neurosensory complications of IAN in Khullar et $\mathrm{al}^{9}$ study which is to some extent in line with the findings of the present study, although the difference of the experimental and control groups' parameters in the two studies are not consistent.

Besides, Miloro and Repasky ${ }^{12}$ focused on the possible effects of low-level laser irradiation before and after treatment on the recovery of neurosensory disorders resulting from BSSO according to the subjective as well as the objective criteria. Their findings demonstrated that as a result of low-level laser irradiation as an adjuvant therapy, improvements of neurosensory disorders following the bilateral BSSO surgery was significant regarding the time and magnitude of return of function based on both subjective and objective criteria which are supported by the obtained results of the present study as well.

In addition, Gasperini et $\mathrm{al}^{13}$ in their study addressed the results of the low-level laser irradiation in improving the neurosensory disorders caused by sagittal split osteotomy of mandibular. They observed significant differences between the two sides with respect to the results of two-point discrimination test in the chin skin; furthermore, the contact test showed a significant difference between two sides. Thirty days after the surgery, significant differences were noticed between the groups in the chin skin sensitivity and the results of the twopoint discrimination test. In their study, the difference between the sides with and without the treatment in the two-point discrimination test increased 60 days after the surgery and these differences were detected to be significant with respect to the chin skin sensitivity. The researchers showed that low-level laser irradiation was able to accelerate the recovery of tissue reactions and reversibility of neurosensory disorders following BSSO surgery. In contrast, the values of two-point discrimination test of the present study were slightly higher in the control side compared with those of the experimental side, and the observed differences were not statistically significant.

In another study conducted by Ladalardo et $\mathrm{al}^{14}{ }^{14}$ clinical cases of the peripheral nerve lesions were selected and the effects of diode laser in the treatment of lip paresthesia after the surgery were focused on. Patients suffered from a number of complications, such as lack of the sense of taste, decrease in the sensitivity, and an increase in the threshold receptors in the tongue. According to the results of the conducted study, the laser irradiation was beneficial in returning the sensitivity of all six patients.

However, it is worth mentioning that such studies do not present sufficient information about the parameters of low-level laser irradiation in general and their dose 
in particular. ${ }^{15}$ If there is no standard for laser beam calculations, dose calculations, and their reporting style, some problems may occur in the reproducibility of the laser-irradiation method, and the clinical results of these studies will not be consistent with one another.

The mechanism of low-level laser performance in improving neurosensory disorders associated with surgeries, such as BSSO has not been precisely specified yet; however, it seems that laser at specific wavelengths of 830 to $820 \mu \mathrm{m}$ penetrates to the axons tissues, Schwann cell, and around the damaged nerve sites, and perhaps the effects of low-level laser can take place at the cellular metabolic levels resulting from the stimulation of fibers and light-sensitive enzymes (rhodopsin-kinase-type enzymes) in the damaged axons. Damaged nerve areas are stimulated following laser irradiation and produce specific proteins which may contribute to the nerve regeneration. It has been indicated that human neuroblastoma cell lines following low-level laser treatments has shown reductions of lowry protein in the laser-treated cells which specifies that these cells actively combine some proteins. ${ }^{16}$ On the contrary, some theories show that undamaged axons and Schwann cells in the damaged axon areas experience upregulation; hence, strengthening the regeneration of damaged axons is done through the production of neurotrophic and neurotropic factors. These undamaged axons are then involved in neurotransmission of the target site's tissues, which has already been done by the damaged axons. Schwann cells play an essential role in the nerve regeneration through the production of trophic factors ${ }^{17}$ and provide physiological channels for regeneration of the damaged axons; their sensitivity to the stimulation caused by low-level laser certainly supports this hypothesis. The area involving neural and metabolic effects of low-level laser therapy, of course, requires further evaluations.

In low-level laser irradiation, laser protocol selection must be done with due care as laser irradiation energy may have diverse effects, and the change in some parameters of laser irradiation leads to the changes in the results of the treatment. Lanzafame et $\mathrm{al}{ }^{18}$ and Lopes et $\mathrm{a} 1^{19}$ have conducted studies with the same amounts of laser energy and different power density; however, they have reported absolutely different results.

At present, there is no ideal low-level laser protocol for the treatment of neurosensory disorders resulting from BSSO surgery. In Khullar et $\mathrm{al}^{9}$ study, the $4 \times 6 \mathrm{~J}$ protocol was employed in the treatment of IAN disorders and in one-third areas of lateral lower lip, inner cavity and buccal apex of the 2nd molar tooth, apex of the 2nd molar tooth, and lingual of mandibular foramen (total of 20 sessions). ${ }^{11}$ In Miloro and Repasky's ${ }^{12}$ research, the actual laser protocol $(4 \times 6 \mathrm{~J}$ for each session) was used for the IAN in four positions (a total of seven sessions). Various protocols are taken advantage of in other studies which have offered acceptable results. ${ }^{20,21}$

\section{CONCLUSION}

Low-level laser irradiation following the BSSO surgery has presented beneficial effects on improving patient's neurosensory functions with respect to the recovery time and magnitude of function, although no significant difference was observed between the experimental and control groups in some tests.

\section{REFERENCES}

1. Høgevold HF, Trumpy IG, Skjelkbred P, Lyberg T. Extraoral subcondylar ramus osteotomy for correction of mandibular prognathism. J Craniomaxillofac Surg 1991 Nov;19(8):341-345.

2. Cunningham LL, Tiner BD, Clark GM, Bays RA, Keeling SD, Rugh JD. A comparison of questionnaire versus monofilament assessment of neurosensory deficit. J Oral Maxillofac Surg 1996 Apr;54(4):454-459.

3. Zuniga JR, Meyer RA, Gregg JM, Miloro M, Davis LE. The accuracy of clinical neurosensory testing for nerve injury diagnosis. J Oral Maxillofac Surg 1998 Jan;56(1):2-8.

4. Karas ND, Boyd SB, Sinn DP. Recovery of neurosensory function following orthognathic surgery. J Oral Maxillofac Surg 1990 Feb;48(2):124-134.

5. Fridrich KL, Holton TJ, Pansegrau KJ, Buckley MJ. Neurosensory recovery following the mandibular bilateral sagittal split osteotomy. J Oral Maxillofac Surg 1995 Nov;53(11):1300-1306.

6. Paulis GW, Steinhauser EW. A comparative study of wire osteosynthesis versus bone screws in the treatment of mandibular prognathism. Oral Surg Oral Med Oral Pathol 1982 Jul;54(1):2-6.

7. Nishioka GJ, Zysset MK, Van Sickels JE. Neurosensory disturbance with rigid fixation of the bilateral sagittal split osteotomy. J Oral Maxillofac Surg 1987 Jan;45(1):20-26.

8. Midamba ED, Haanes HR. Low reactive-level $830 \mathrm{~nm}$ GaA1As diode laser therapy (LLLT) successfully accelerates regeneration of peripheral nerves in human. Laser Ther 1993;5:125-129.

9. Khullar SM, Emami B, Westermark A, Haanaes HR. Effect of low-level laser treatment on neurosensory deficits subsequent to sagittal split ramus osteotomy. Oral Surg Oral Med Oral Pathol Oral Radiol Endod 1996 Aug;82(2):132-138.

10. Reddy GK. Photobiological basis and clinical role of lowintensity lasers in biology and medicine. J Clin Laser Med Surg 2004 Apr;22(2):141-150.

11. Khullar SM, Brodin P, Barkvoll P, Haanes HR. Preliminary study of low-level laser for treatment of long-standing sensory aberrations in the inferior alveolar nerve. J Oral Maxillofac Surg 1996 Jan;54(1):2-7.

12. Miloro M, Repasky M. Low-level laser effect on neurosensory recovery after sagittal ramus osteotomy. Oral Surg Oral Med Oral Pathol Radiol Endod 2000 Jan;89(1):12-18.

13. Gasperini G, de Siqueira IC, Costa LR. Lower-level laser therapy improves neurosensory disorders resulting from bilateral mandibular sagittal split osteotomy: a randomized crossover clinical trial. J Craniomaxillofac Surg 2014 Jul;42(5):e130-e133. 
14. Ladalardo TC, Brugnera Junior A, Pinheiro AL, Takamoto M, Campos RA. A low-level laser therapy in treatment of neurosensory deficit following surgical procedures. Prog Biomed Opt Imaging 2001;2:152-156.

15. Jenkins PA, Carrol JD. How to report low-level laser therapy/ photo-medicine dose and beam parameters in clinical and laboratory studies. Photomed Laser Surg 2011 Dec;29(12): 785-787.

16. Gift JJ, Ness GM, Mallery SR, Miloro M. Low-level laser irradiation of human neural and perineural cells in vitro. J Oral Maxillofac Surg 1996;54(Suppl 3):108.

17. Williams LR, Azzam NA, Zalewski AA, Azzam RN. Regenerating axons are not required to induce the formation of a Schwann cell cable in a silicone chamber. Exp Neurol 1993 Mar;120(1):49-59.

18. Lanzafame RJ, Kurtz AF, Connelly R, Peter TA Sr, Brondon P, Olson D. Reciprocity of exposure time and irradiance on energy density during photo-radiation on wound healing in a murine pressure ulcer model. Laser Surg Med 2007 Jul;39(6):534-542.

19. Lopes NN, Plapler H, Chavantes MC, Lalla RV, Yoshimura EM, Alves MT. Cyclooxygenase-2 and vascular endothelial growth factor expression. In 5-fluorouracilinduced oral mucositis in hamsters: evaluation of two low-intensity lasers protocols. Support Care Cancer 2009 Nov;17(11):1409-1415.

20. Enwemeka CS, Parker JC, Harkness EE, Harknes LE, Woodruff LD. Efficacy of low-power lasers in tissue repair and pain control: a meta-analysis study. Photomed Laser Surg 2004 Aug;22(4):323-329.

21. Suzuki S, Itoh K, Ohyama K. An in vitro experimental model for studying wound-healing after laser irradiation in the mouse foetus. J Craniomaxillofac Surg 2004 Aug;32(4): 193-198. 